\title{
IR microscope measurement of the plasma spreading in thyristors
}

\author{
by B. Więcek, A. Michalski and K. Napiórkowski
}

Technical University of $Ł o d z$, Institute of Electronics, Computer Thermography Group

18/22 Stefanowskiego St, 90-924 Łódź, Poland, e-mail: wiecek@ck-sg.p.lodz.pl

\section{Abstract}

Plasma spreading and development of conductive path during turn-on process in today's highcurrent thyristors, is one of the main limits to the use of these power devices in high-speed applications. There are a few methods of measurement of dynamic parameters in thyristors. Recombination radiation caused by the current in the semiconductor is measured with the use of a contactless IR method. We propose to use an IR microscope with the detector of the recombination radiation in silicon $(\lambda \approx 1.14 \mu \mathrm{m})$.

\section{Theoretical and experimental aspects}

Plasma spreading rate during turn-on process is one of the dynamic parameter of thyristors. Development of conductive path is mainly caused by the gradient of minority carriers (they are in the whole structure of thyristor especially in the p-base and the n-base) and by the transverse electric field (the largest in the transition area).

Rate of the development depends on a few parameters:

- current's density,

- transverse electric field,

- doping level,

- lifetime of the minority carriers in n-base,

- emitter's short.

The mechanism of plasma spreading is based on the diffusion of majority carriers and carrying the carriers by strong electric field. Diffusion depends on the gradient of the carriers' concentration, which is function of current density in the conductive path. Greater current density causes the increase of the rate of plasma spreading. Experimental researches leaded to many equations. One of them is shown below:

$$
V=C J^{\frac{1}{n}}
$$

where: $C$ and $n=2-6$ are constants.

The transverse electric field takes place in both p-base and n-base. It is mainly located in transition area, on the border of conductive and non-conductive area. The transition area is narrow. It is also known that its width is about a few diffusion lengths. This means that, when the structure partly conducts and the contact voltage is about few Volts, the transverse electric field near the collector junction can achieve $10^{3} \mathrm{~V} / \mathrm{cm}$. The rate of plasma spreading as a function of current's density can be put in the form:

$$
V=A \ln (J)+B
$$

where: $A$ and $B$ are constants.

The doping level and width of $p$-base influence the four-layer structure of current gain coefficient of $n-p-n$ transistor. When the doping level increases, the gain, rate of plasma spreading and depth of positive feedback, decreases $\left(\alpha_{1}+\alpha_{1} \rightarrow 1\right)$. Experimental investigations reveal that a doping level of about $N_{A}=10^{17}-10^{18} \mathrm{~cm}^{-3}$ causes a reduction of the plasma spreading from $500 \mathrm{~m} / \mathrm{s}$ to $50 \mathrm{~m} / \mathrm{s}$ (for a current density of about $400 \mathrm{~A} / \mathrm{cm}^{2}$ ). 


$$
V \sim N_{A}^{-0.9}
$$

The rate of plasma spreading depends also on the width of the p-base and n-base. When the width of the base ( $p$-base or $n$-base) increases, the coefficient of current gain increases too $\left(\alpha_{1}, \alpha_{1}\right)$. Experimental equation for the structure with the doping of gold at a temperature of $860^{\circ} \mathrm{C}$ is shown below (emitter is shorted, current's density $\mathrm{J}=100 \mathrm{~A} / \mathrm{cm}^{2}$ ).

$$
V \sim W_{B n}^{-l}
$$

where: $l \approx 1$.

When we consider the lifetime of the minority carriers in $n$-base $\left(V \sim \tau_{p}{ }^{1 / 2}\right)$, we get to the conclusion:

$$
V \sim \frac{\tau_{p}^{1 / 2}}{W_{B n}} \sim \frac{L}{W_{B n}}
$$

where: $L$ means the way of diffusion in the $n$-base.

Similar conclusion and equation can be obtained for the lifetime of minority carriers and the width of $p$-base.

Changing the current gain of $n-p-n$ transistor causes influence of emitter's short on plasma spreading. The coefficient of "density of emitter shorts" is described by the diameter of these shorts (d) and their clearances (D). Increasing "density of emitter shorts" leads to decreasing of the rate of plasma spreading.

$$
\ln (V) \sim \frac{1}{16}\left(d^{2}+D^{2}\left[2 \ln \left(\frac{D}{d}\right)-1\right]\right)
$$

The plasma spreading depends on the current gains of both transistors, which are part of thyristor. This dependence is shown below:

$$
V \sim \ln \left(\alpha_{1} \alpha_{2}\right)
$$

\section{Detection of recombination radiation}

In order to detect the recombination radiation, a wide-band detector with a narrow bandpass $\mathbb{R}$ filter or detector with narrow spectral characteristic (maximum sensitivity for $\lambda \approx 0.91 \div 1.2 \mu \mathrm{m})$, has to be used.

We propose to use IR microscope with the detector of recombination radiation in silicon $(\lambda \approx 1.14 \mu \mathrm{m})$ - Fig. 1 . The measurement requires the removal of the metal from the upper surface of thyristor. If the structure is symmetric we remove a narrow radial path of metal of about $500 \mu \mathrm{m}$ thickness. We have used IR Microscope Barnes RM-2A equipped with a silicon diode to absorb infrared radiation at the room temperature of $294 \mathrm{~K}$. The optical system of the microscope (reverberate objective) asserts pixel detection of recombination radiation Fig. 2.

\section{Results}

We have made by investigations measuring the output signal of the detector of recombination radiation in five different points of thyristor's structure. These points were located along the radius of the thyristor (Fig. 3), at different distances from its center:

The radius of the thyristor was $r_{\text {max }}=0.78 \mathrm{~cm}$. The measurements were made for current impulses (half-wave sinusoid) with amplitude $I_{T M}=100 A \div 500 A$ and duration of $t_{i T}=50 \div 275 \mu$ s (Fig. 4). It was assumed that the border of conductive area is a circle (for the geometric symmetry of the structure and uniform plasma spreading). Examples are shown in Fig. 5. 
The beginning of the diagram denotes the moment of thyristor turn on. The basic model of the plasma spreading rate was appointed by taking under consideration the temporary current density. This model can be described by:

$$
V=A \cdot \ln (J)+B
$$

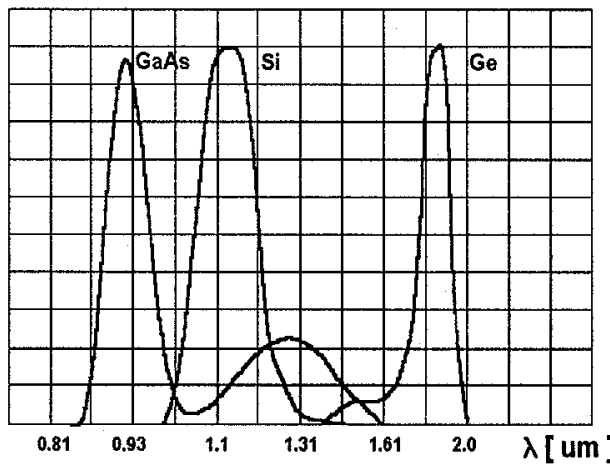

Fig. 1. Recombination radiation for different semiconductors in room temperature

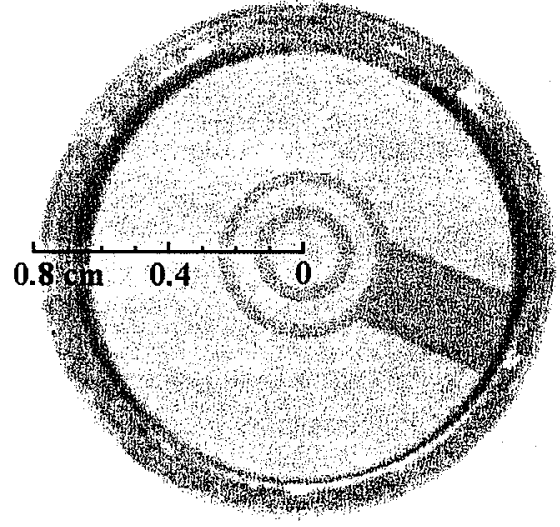

Fig. 3. Amplifying gate thyristor $I_{A V}=40 \mathrm{~A}$

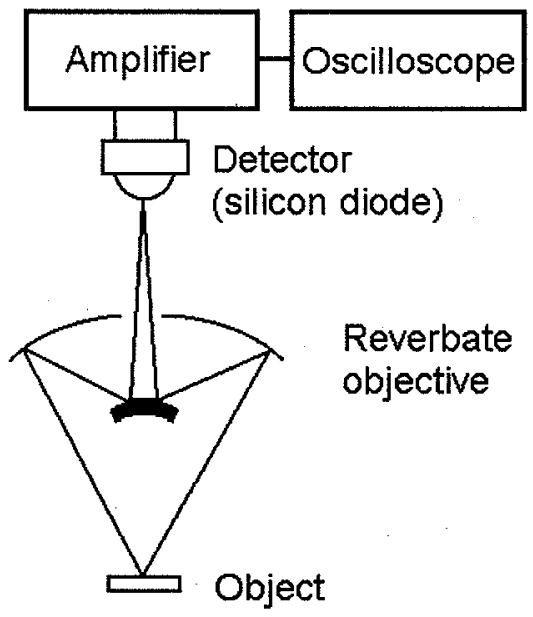

Fig. 2. System setup

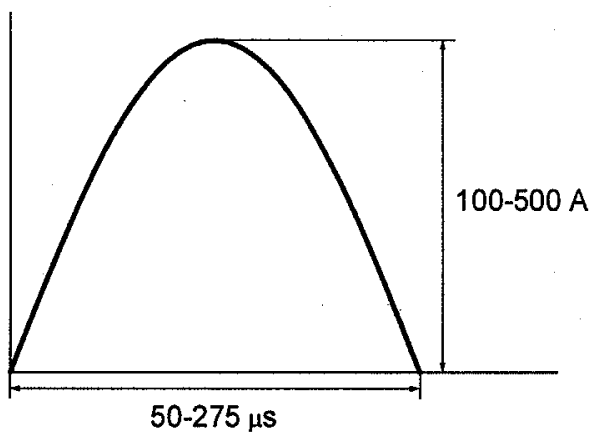

Fig. 4. The current excitation 


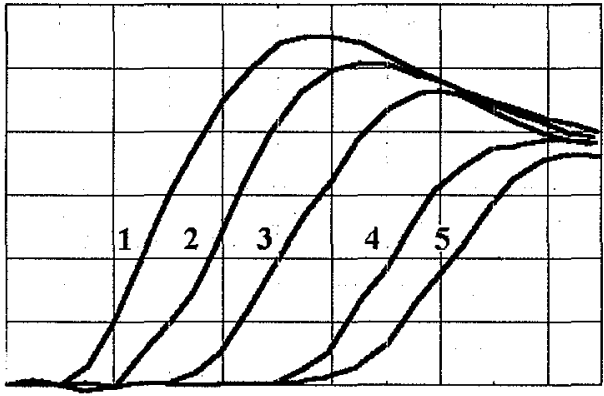

Fig. 5. Intensities of the recombination radiation in different points on the radius of the thyristor

$$
\left(I_{T M}=200 A, t_{i M}=275 \mu s, 10 \mu s / d i v\right)
$$

$1-0.30 \mathrm{~cm} ; 2-0.35 \mathrm{~cm} ; 3-0.40 \mathrm{~cm}$;

$$
4-0.45 \mathrm{~cm} 5-0.50 \mathrm{~cm}
$$

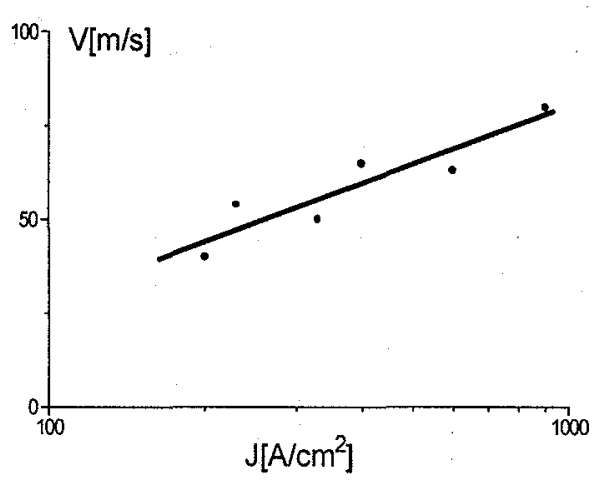

Fig. 6. Plasma spreading rate versus current density (experimental results)

The obtained results show the agreement of measurements with the theoretical considerations. We have confirmed the basic dependence of plasma spreading rate and current density in thyristors. The logarithmic characteristic $V=A \ln (J)+B$ is the proper approximation of the development of conductive channel in the device. Experimental data can be used in the modeling of electrical and thermal phenomenon in microelectronic power components. The rate of plasma spreading is one of the key parameter and is significant on the power dissipation and on the temperature distribution in a power device. We have made several thermo diagrams showing the temperature versus the size of the conductive path in thyristors. These investigations will be continued in order to provide an improvement of the technological parameters in high power, high temperature devices.

\section{REFERENCES}

[1] RUHL (H.) - Spreading Velocity of the Active Area Boundary in a Thyristor. IEEE Trans. ED, Vol.17, No.9, Spetember 1970.

[2] SAWAKI (N.) - High Field and Lateral Current at Turn-on of Large Area Thyristor. Japanese Journal of Applied Physics, Vol. 16, Suplement 16-1,1987.

[3] TERASAWA (Y.), FUKUI (H.) - Calculation of Two-Dimensional Temperature rise Distribution in a Thyristor. IEEE Trans. ED, August 1976.

[4] WIEcCEK (B.), GRECKI (M.), PACHOLIK (J.) - Computer-based thermographic system. QIRT'92 - Eurotherm Series No. 27 - EETI ed., Paris 1992. 